\title{
Hypertension: a problem of organ blood flow supply-demand mismatch
}

\author{
Maarten P Koeners ${ }^{*, 1}$, Kirsty E Lewis' ${ }^{1}$ Anthony P Ford ${ }^{2}$ \& Julian FR Paton'
}

This review introduces a new hypothesis that sympathetically mediated hypertensive diseases are caused, in the most part, by the activation of visceral afferent systems that are connected to neural circuits generating sympathetic activity. We consider how organ hypoperfusion and blood flow supply-demand mismatch might lead to both sensory hyper-reflexia and aberrant afferent tonicity. We discuss how this may drive sympatho-excitatory-positive feedback and extend across multiple organs initiating, or at least amplifying, sympathetic hyperactivity. The latter, in turn, compounds the challenge to sufficient organ blood flow through heightened vasoconstriction that both maintains and exacerbates hypertension.

First draft submitted: 10 July 2015; Accepted for publication: 10 March 2016;

Published online: 19 April 2016

\section{Background}

Causes of elevated sympathetic activity in neurogenic hypertension remain enigmatic. We explore evidence to support the notion of blood flow supply-demand mismatch to an organ as a factor involved in the initiation of raised sympathetic activity, and its subsequent amplification, in hypertension. We first consider some homeostatic mechanisms regulating blood flow and then discuss emerging changes that may occur during the development of hypertension that sensitize afferent innervation of target organs. We hypothesize that activation of visceral afferent systems induces increases in sympathetic nerve activity (SNA) that can initiate and sustain hypertension. We term this the visceral afferent activation hypothesis of hypertension.

\section{- Physiological mechanisms ensuring organ blood flow homeostasis: a brief overview}

All organs have a capacity to increase blood flow through local functional (reactive) hyperemia, autoregulatory, endocrine and neural mechanisms. Blood flow autoregulation is defined as the ability of an organ to maintain a constant blood flow despite either increases or decreases in blood (perfusion) pressure. Blood flow autoregulation is intrinsic to the arterioles within the organ and occurs in the absence of neural and hormonal influences. Endocrine controls include epinephrine and norepinephrine, as well as vasopressin, the renin-angiotensin-aldosterone system, atrial natriuretic peptide and erythropoietin. Neural mechanisms include baroreceptors in the aorta, carotid sinuses and right atrium, and peripheral and central chemoreceptors that monitor blood levels of oxygen, carbon dioxide and hydrogen ions; together these systems modulate levels of autonomic and hormonal activity through central reflex arcs coursing through various interconnected CNS

\footnotetext{
'School of Physiology, Pharmacology \& Neuroscience, Biomedical Sciences, University of Bristol, Bristol, UK

${ }^{2}$ Afferent Pharmaceuticals, 2929 Campus Drive, San Mateo, CA, USA

*Author for correspondence: Tel.: +44 011733 11629; m.koeners@bristol.ac.uk
}

\section{Future CARDIOLOGY}

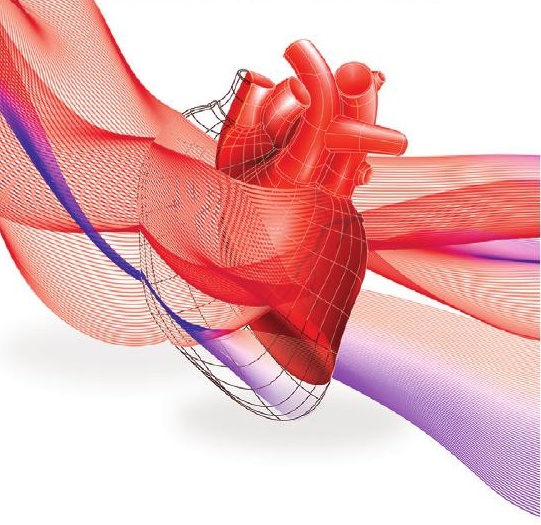

KEYWORDS

- hypertension

- hypoperfusion • organ

blood flow $\bullet$ sympathetic

hyperactivity $\bullet$ visceral

afferent hyper-reflexia 
structures. Such structures also bring about blood flow homeostasis during stress, hyperosmolarity, emotion, fear and anxiety. In physiological conditions, the aforementioned homeostatic mechanisms will distribute blood flow, and hence oxygen, to organs in proportion to their metabolic demand, which is precisely controlled [1]. Normally, oxygen delivery exceeds the rate of oxygen consumption (demand) safeguarding capacity within each organ, which can be tapped into as a change in state demands [1]. For example, upon ascending to high altitude or during exercise, blood flow is increased to maintain a preferred supply/demand ratio [1] However, if demand outstrips supply (i.e., a blood flow supply-demand mismatch), then hypoxic-hypoperfusion results. This might be corrected by increasing perfusion pressure, perhaps evoked through excitation of the organ's own afferent system. However, according to Poiseuille's Law, at constant perfusion pressure blood flow is directly proportional to the fourth power of the radius of the vessel, meaning that a proportionally large increase in blood pressure will be needed to restore blood flow if the radius decreases, that is, the resistance increases. Blood pressure can be elevated by increasing either cardiac output and/or vascular resistance through activation of a neurohumoral mechanism including vasomotor sympathetic activity. Once the organ's oxygen demand returns to basal levels, hemodynamic homeostasis is restored. However, if organ hypoxic hypoperfusion persists, or if the afferent circuits from that organ convey signals of such condition, even if aberrantly once it has passed, we surmise that this may result in augmented sympathetic discharge and, thus, hypertension. It has been maintained that most forms of hypertension are associated with increased total peripheral resistance (TPR), rather than increased cardiac output [2]. In such conditions, increased SNA in both men and older women is a primary driver of increased TPR [3]. Chronic elevation of TPR as a mechanism to increase blood pressure may reduce organ blood flow capacity, thereby increasing the vulnerability to tissue hypoxic-hypoperfusion. Since all organs are innervated by sensory nerves, we propose that organ hypoperfusion will activate afferent activity and reflexly stimulate sympathetic hyperactivity leading to hypertension. However, this generalized vasoconstriction may compromise blood flow to other organs and thus trigger blood flow a broad supply-demand mismatch, which may result in the recruitment of additional afferent drivers that compound the hypertensive state. We explore this cycle of maladaptation in more detail giving examples to substantiate this notion.

The sympathetic problem of hypertension Unequivocal evidence supports chronic activation of the sympathetic nervous system as a characteristic of hypertension and its participation in the initiation, maintenance and progression of blood pressure in experimental animal and human studies [4]. A big clinical challenge is to unveil the cause(s) of sympathetic overactivation and design novel therapies that lower it below its threshold for triggering significant increases in TPR. Evidently, sympathetic hyperactivity interacts with many pathologies beyond neurogenic hypertension, including heart failure, salt sensitivity, obesity, glucose intolerance, rheumatoid arthritis and many more. However, the aim of our review is to understand the relationship between organ hypoperfusion and subsequent visceral afferent activation as a potential driver of sympathetically mediated hypertension.

\section{Visceral afferent hyperactivity: consequences for sympathetic activity \& organ blood flow}

To the best of our knowledge, all organs are connected to the brain reciprocally by visceral afferent and efferent nerves (Figure 1). Many of these afferent nerves can trigger sympathoexcitatory responses through spinal and supraspinal reflexes. Exceptions include baroreceptors, right atrial and pulmonary stretch receptors and some renal afferents that inhibit sympathetic activity and may oppose hypertension-induced sympathetic hyperactivity [5]. Interestingly, these receptors are mechanosensory and deactivated by hypoperfusion and/or metabolic stimuli so may not be relevant to blood flow supply-demand mismatch [5]. We propose that metabolite-sensitive afferents (metabosensors) that trigger sympathetic activity responses are less susceptible to desensitization (adaptation) and, under pathophysiological conditions can: become sensitized, producing exaggerated reflex responses (hyper-reflexia); and generate aberrant tone (hypertonicity); the latter has the potential to provide a sustained drive for maintaining high levels of sympathetic activity (Figure 1). Their activation may actively depress sympatho-inhibitory reflexes, such as the arterial baroreceptor reflex. 


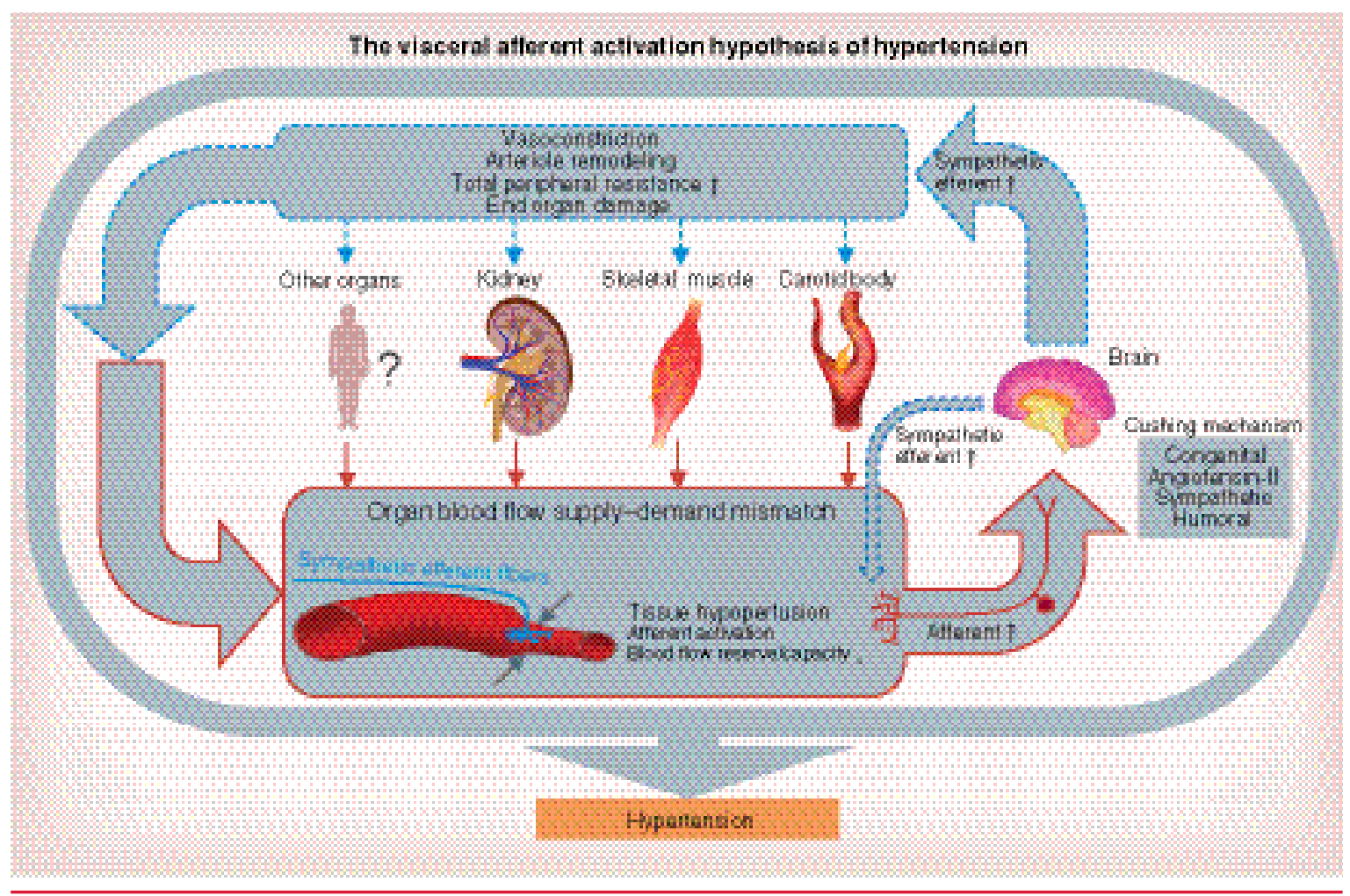

Figure 1. Visceral afferent activation hypothesis of hypertension. When afferents become sensitized due to, for example, high sympathetic activity, circulating angiotensin II, atheroma or congenital predisposition (although exact mediator[s] remain to be identified) they reflexly increase sympathetic vasomotor tone reducing making an organ vulnerable to hypoxic-hypoperfusion and reducing its blood flow reserve/capacity. We propose that this triggers release of metabolites that activate excessive afferent nerve activity, which exacerbate the reflexevoked sympathetic vasoconstriction. Widespread sympathetic activation may recruit additional afferent systems resulting in additional drivers for maintaining pathologically high levels of sympathetic activity and total peripheral resistance. Thus, the system degenerates into a positive feedback loop in hypoxic-hypoperfusion and reduced blood flow reserve/capacity acts as an amplifier and conceivable initiator of sympatho-excitatory afferent drive. Consequently, vasoconstriction, arteriole remodeling increased total peripheral resistance, and end organ damage will amplify the organ blood flow supply-demand mismatch compounding the problem of hypoperfusion and the development and maintenance of hypertension. We do not rule out that excessive sympathetic activity itself sensitizes the afferent nerves mediating the reflex-evoked sympatho-excitation.

Artwork was provided by Michel Cekalovic [12].

Evidence supporting the presence of excessive afferent signaling in hypertension comes from the kidney [6,7] and carotid body [8-11] as their selective denervation can reduce both sympathetic activity and blood pressure, as we discuss later.

It is important to recognize that physiological afferent signaling from an organ may occur in response to stimulants released locally because of a change in metabolic demand. Such examples include: reduced blood flow and hypoxia, hypercapnia and metabolic stress. Functional or reactive hyperemia in skeletal muscle is a mechanism that equates the change in metabolic demand with increases in blood flow. The metabolites leached from respiring tissues cause localized vasodilatation but, at sufficient concentration, also stimulate afferent endings (group III or IV fibers). The latter results in reflex increase in sympathetic activity and arterial pressure, with the sole aim of increasing perfusion to the muscle bed from which the afferents were activated. Functional hyperemia normally offsets sympathetically mediated vasoconstriction [13]. The net result is elevated blood flow to the metabolically active organ to satiate its increased demand for oxygen. The increased perfusion also serves to washout metabolites, reduce their accumulation and may temper afferent activity to a level that optimizes oxygen supply and demand; hence an 
optimal equilibrium is reached. Afferent signaling normally ceases once oxygen supply outstrips demand. However, in disease states, such as hypertension, afferent signaling may become exaggerated and persist even in the absence of metabolites. Further, local metabolites mediating the vasodilatation may be less effective at opposing high levels of sympathetically mediated vasoconstriction [14,15]. Indeed, in hypertension, nitric oxide did not attenuate sympathetic vasoconstriction during exercise - an effect seen in animals with normal blood pressure [14]. We discuss these possibilities below.

\section{- Sensitization of afferent feedback generating sympathetic excess in hypertension}

Sympathetic activation patterns may be graded and/or differentially controlled on an organ basis. For example, the classic 'defense' or 'alerting' response is characterized by increases in arterial pressure and heart rate with vasodilatation in skeletal muscle (mediated, in part, by sympathoinhibition), and vasoconstriction in the splanchnic, renal and cutaneous vascular beds [16]. Our notion is that in conditions of hypertension there is an underlying imbalance, with vasodilatory metabolites giving way to predominance of sympathetic vasoconstrictor influence that is driven by afferent sensitization and emergence of aberrant afferent tonicity. The mechanism(s) involved in afferent sensitization and tone generation remain an open question and may be organ and state dependent but could result as a consequence of hypoxic-hypoperfusion triggering transcriptional processes. We propose that a plethora of initiators/mediators could be involved in this sensitization, including metabolic stressors (hypoxia, hypercapnia, hyperglycemia, ATP, potassium ions, low $\mathrm{pH}$ ), oxidative stress, anatomical anomalies (preconditioned, congenital), anemia, respiratory stress, atherosclerotic plaque, inflammation and renin-angiotensin system (RAS) activation. These mediator(s) themselves may sensitize the very mechanisms that cause depolarization and trigger receptor potentials in afferent endings resulting in a chronic excitatory effect. Important to our hypothesis is that aberrant afferent firing persists at rest in the absence of any metabolic stimuli and, thus, is a pathological signal. The latter is highly relevant as at rest there will be an absence of opposing vasodilatory metabolites making sympathetically mediated vasoconstriction more intense, worsening the problems of hypoperfusion and hypertension. Through a vicious circle, the latter would result in further stimulation of afferent activity and reflexly evoked sympathetic vasoconstriction accentuating the problem of blood flow supply-demand mismatch. We do not rule out a crosstalk mechanism, whereby sympathetic activity itself sensitizes adjacent afferent endings as an additional amplifying mechanism; there is at least anatomical evidence for this in the kidney where afferents and sympathetic fibers are intertwined [17]. Highly analogous is somatosensory sensitization seen in states of 'sympathetically maintained pain' or reflex sympathetic dystrophy, now thought of as 'complex regional pain syndrome'. These are chronic pain conditions associated with sympathetic sprouting onto primary afferents terminals, dorsal root ganglion and spinal dorsal horn afferent terminals [18]. Being released by sympathetic postganglionic neurones, ATP participates in this sensitization [19]. We propose that the same process of sensitization may apply to visceral afferent activation of hypertension.

Heightened activation of the sympathetic nervous system through aberrant afferent tonicity from an organ may offset the vasodilatation produced by functional hyperemia. For example, abnormal muscle afferent signaling in heart failure patients increased the passive leg movement-induced increases in norepinephrine spillover and arterial blood pressure, which significantly reduced femoral blood flow, oxygen delivery and tissue oxygen saturation [20]. If sympathetically mediated vasoconstriction becomes widespread across vascular beds this may recruit multiple sympatho-excitatory afferent systems and amplify the problem of raised blood pressure (Figure 1). Additionally, autoregulation in response to the rising perfusion pressure will also increase vascular resistance in an attempt to maintain constant blood flow, but at the expense of further elevating TPR. Moreover, persistent and heightened sympathetic activity may reset autoregulation to higher pressure levels (e.g., renal blood flow autoregulation curve is shifted to a higher range of renal arterial pressures in hypertensive dogs with enhanced sympathetic activity [21]), as well as cause arteriolar remodeling [22]; this would have deleterious consequences for blood flow supply-demand matching. Finally, it is also likely that hypoxichypoperfusion and reduced blood flow become greatly agonized at night when blood pressure 
normally dips [23]. In a constant state of sympathetic overactivity, it is easy to see how nocturnal dipping is lost in conditions of hypertension [24]. Below we consider some examples of organs exhibiting excessive afferent activity and heightened SNA in hypertension.

Potential sources of afferent hyperactivity

Although it seems that many, if not all, organs affect blood pressure regulation, we will focus within this review on the organs that we consider as exemplary of our hypothesis and where evidence exists supporting organ hypoperfusion and blood flow supply-demand mismatch triggering dysfunctional afferent activity and reflexly driven sympathetic activity.

\section{- Kidney afferents \& hypertension}

Renal afferent nerves primarily terminate peripherally in the renal pelvis and project to the spinal cord with onward transmission to the nucleus tractus solitarii, rostral ventrolateral medulla (RVLM), subfornical organ and paraventricular nucleus (PVN). There are single modality fibers (mechanoreceptors) that sense stretch of the pelvic wall causing sympatho-inhibition, chemosensitive receptors causing sympatho-activation and multimodal fibers that sense both (reviewed in [25]). Neurally mediated responses occurring in one kidney as a result of interventions on the same or opposite kidney have been defined as renorenal reflexes, which function to decrease renal efferent SNA to minimize sodium retention [26]. However, in ischemic/hypoxic kidneys and/or in the hypertensive state, there is a reversal of the renorenal reflex: stimulation of afferent nerves by ischemic metabolites, such as adenosine, and/or by uremic toxins, such as urea [27], which enhances the sympatho-excitatory state, increases salt retention and blood pressure. This reversal of the renorenal reflex is significant since afferent signals emanating from the kidney to the CNS have been strongly associated with the etiology of hypertension. Indeed in experimental animal models of hypertension, the removal of the afferents by either dorsal rhizotomy [7,28-30] or specific inhibition of afferent fibers by capsaicin treatment [6] have proven to be antihypertensive. Interestingly, Cowley et al. [31] has demonstrated that a shift in the redox balance and oxidative stress reduces the blood flow to the medulla, a mildly hypoxic area of the kidney, leading to hypertension and renal injury. This supports the concept of oxidative stress as an initiator/mediator involved in hypertension, potentially via hypoxia-hypoperfusion and afferent sensitization. Recent clinical trials have shown that blood pressure and SNA are both reduced after bilateral denervation (afferent and efferents) of the kidneys in some drug-resistant hypertensive patients [32-34], although the efficacy of this intervention is debated [35]. Recently, it has emerged that afferents are important for the hypertensive state in chronic kidney disease [36]. Future studies are needed to understand the mechanisms for renal afferent sensitization and tonicity in hypertensive patients; putative stimulants are discussed below. Hypothetically, understanding the latter may provide an effective means to select patients most likely to respond to treatment (pharmacological or denervation). Taken together, these data emphasize the role of afferent signals emanating from the kidney in the generation of hypertension.

In recent years, evidence has accumulated that kidney hypoxia plays a significant role in the pathogenesis and progression of renal disease [37-40]. Even a small lesion in the kidney, resulting in an area(s) of ischemia not necessarily affecting renal function, can cause hypertension, perhaps via afferent activation [41]. Renal hypoperfusion, ischemia and hypoxia can all trigger afferent signaling to reflexly elevate sympathetic activity and arterial pressure. This has led to the prediction that renal hypoxia is not just a consequence of kidney disease, but rather a primary pathogenic event [37-39,42-48]. But what could be the initial trigger? Exogenous administration of angiotensin II (AngII) can lead to hypoperfusion of postglomerular peritubular capillaries, and subsequent hypoxia within the tubules and interstitial space $[40,49]$. We propose that hypertensive kidney disease is driven by vicious loops of positive feedback, initiated by increased AngII triggered by hypoperfusion and accompanied by nephron loss, inflammation and diabetes. This results in oxidative stress and nitric oxide deficiency augmenting hypoperfusion and renal afferent signaling. Therefore, improving blood perfusion and thus oxygenation of the kidneys may reduce SNA and arterial pressure and improve renal function, thereby providing a novel future treatment strategy. This is supported by the findings that: pharmacologically increasing renal oxygen consumption per se caused renal hypoxia, proteinuria [50] and eventually leads to renal injury [51] and; application of $100 \%$ oxygen to patients with chronic kidney 
disease reduced muscle SNA, whereas such an intervention did not affect muscle SNA in control patients [52]. This mismatch in renal oxygen demand and supply exemplifies how afferent driven sympatho-excitatory is a powerful stimulus for hypertension.

\section{- Carotid body afferents \& hypertension}

Stimulation of the carotid body (CB), the dominant peripheral chemoreceptor, drives sympathetic tone through relatively direct signaling to the nucleus tractus solitarii, rostral ventrolateral medulla and paraventricular nucleus resulting in increased blood pressure [53,54]. In cardiovascular diseases, it appears that the CB generates aberrant afferent discharge [8,9]. Schultz et al. have clearly implicated a significant role of CB afferent drive in the sympathetic hyperactivity and breathing dysregulation in animal models of chronic heart failure [55,56]. Blood flow to the $\mathrm{CB}$ is exceptionally high in relation to tissue mass in health [57], but in rabbits with pacinginduced chronic heart failure this was reduced due to lowered cardiac output [56]. This might account for $\mathrm{CB}$ afferent tonicity. Given this, one might predict that lowering $\mathrm{CB}$ afferent drive should be therapeutically beneficial. As a proof of principle, $\mathrm{CB}$ resection was performed in heart failure patients and partially corrected cardiac autonomic balance and cardiac baroreflex gain $[10,11]$ and reduced muscle sympathetic activity. Moreover, the CB is an afferent source driving sympathetically mediated hypertension in the spontaneously hypertensive rat (SHR) [8,9]. Again CB chemoreceptors are tonically active in the SHR $[8,9]$ and their disconnection from the brain is antihypertensive, substantially reduces renal sympathetic activity, and improved both baroreflex and renal function [8,9]. Intriguingly, there was an additive blood-pressure lowering effect when renal denervation was subsequently performed [9]. These data strongly support our hypothesis that organ hypoperfusion and blood flow supply-demand mismatch recruits additional sources of afferent drive across organs with each independently contributing to sympathetic excess and hypertension (Figure 1).

\section{- Skeletal muscle afferents \& hypertension} Sympathetic engagement during exercise is initiated, in part, by activation of thin-fiber afferents arising from contracting skeletal muscle. In the case of lower limb muscles, these project via the lumbar dorsal horn of the spinal cord and via supra-spinal circuits modulate cardiovascular and respiratory activity and their reflex control [58]. These afferents are mechanically (mechanoreceptor; type III sensory fibers) and/or metabolically sensitive (metaboreceptor; type IV fibers). During the onset of exercise, these afferents become activated and evoke the 'exercise pressor response' consisting of rises in arterial pressure, heart rate, stroke volume, cardiac output, redistribution of blood flow to active skeletal muscle and hyperventilation [59]. What evidence exists that these afferents become tonically active in the hypertensive state? In hypertension, sensitization of both mechanically and metabolically sensitive skeletal muscle afferents has been demonstrated [60,61]. Moreover, the exercise pressor response is augmented and associated with the development of hypertension [62]. In chronic heart failure, this includes an increased sensitivity of type 3 fibers compared with type 4 and heightened P2X response [63]. Functional sympatholysis, the process whereby sympathetic vasoconstriction is offset by metabolites released from the exercising skeletal muscle, is impaired substantially in hypertension [64]; this results in reduced oxygenation and blood flow in exercising muscles of hypertensive individuals compromising their ability to exercise [64]. This, presumably, leads to persistent afferent activation and hyper-reflexia producing exaggerated pressor responses during exercise but may also contribute to reduced exercise tolerance through fatigue and possibly pain.

There are a wide range of molecules involved in the regulation of blood flow to skeletal muscle during exercise. One major example is ATP. ATP, a potent vasodilator, stimulates the formation of both nitric oxide and prostaglandins [65], which counteract local sympathetic vasoconstriction $[66,67]$. A further source of nitric oxide production is by mechanically induced signals, including shear stress-activated endothelial nitric oxide synthase [68]. Recent studies have shown that oxidative stress further increases vasoconstriction in skeletal muscle in rats with induced hypertension [69-71], suggesting a reduction/absence of the opposing dilatatory influence of nitric oxide. Taken together, this supports our hypothesis of organ hypoperfusion and blood flow supply-demand mismatch and afferent sensitization from a relatively large vascular bed that can drive neurogenic hypertension (Figure 1). 
- Other contributing afferents \& crosstalk Stimulation of intestinal mechanoreceptors (stretch) or chemoreceptors activates splanchnic nerve afferents resulting in decreases in tissue blood flow in the splanchnic organs (duodenum, jejunum, pancreas, spleen, stomach and liver) and kidneys [72]. This is most likely to be mediated by the sympathetic nervous system since electrical stimulation of intestinal afferents increases sympathetic activity in the splanchnic bed [72]. On the other hand, sympatho-inhibition occurs following the ingestion of a meal. This can augment splanchnic blood flow by approximately $150 \%$ above baseline, postprandially [73] . In hypertensive subjects, blood flow to the splanchnic vascular bed is reduced [73], while in obese, hypertensive Sprague-Dawley rats the postprandial sympatho-inhibition and vasodilator effects are abolished. Although this does not separate cause from consequence, it does indicate a connection between splanchnic hypoperfusion, reduced sympatho-inhibition and hypertension.

High blood pressure can induce vascular (arterial/arteriole) hypertrophy [74] as can the increase in sympathetic nervous activity that is associated with hypertension [75-77]. Lumen narrowing, stiffening and exacerbated responses to sympathetic nervous system stimulation may all contribute to elevations in vascular resistance and the hypertensive condition. However, there is also evidence that the arterial remodeling can occur before the development of hypertension as a congenital mechanism in the SHR [78] .

Activation of hepatic afferent nerves causes a decrease in blood flow through the hepatic artery again mediated by sympathetic nerves [79]. Activation of spinal cardiac afferents also increases sympathetic activity within minutes [80] and impairs baroreflex control of renal sympathetic nerve activity in rats [80]. Conceptually, this positive feedback can correct hypoperfusion of cardiac tissue thereby reducing the severity of ischemia and decreasing infarct's size in myocardial ischemia injury. As mentioned above, crosstalk between sympathetic and afferent fibers could be a part of a potential mechanism for sensitization, and afferent aberrant discharge as noradrenaline (NA) can activate sensory receptors directly [17], and ATP is coreleased with NA from sympathetic postganglionic efferents [81] and further activates $C$ fibers. Indeed, there is histological evidence of close coupling between efferent and afferent $C$ fibers in human skin [82] and Kopp et al. have shown that there is a close apposition of afferent and efferent nerves in the renal pelvis [17]. Finally, activation of the sympathetic supply to the CB causing vasoconstriction [83] could also account for its sensitization and aberrant tone in hypertension.

\section{The selfish brain hypothesis of hypertension}

Cushing's response is enunciated as a physiological nervous system response to increased intracranial pressure that results in Cushing's triad of increased blood pressure, irregular breathing and a reduction of heart rate. Paton et al. have proposed that the 'Cushing mechanism' is not just a 'last ditch' protection for critically ischemic brain, but 'a physiological mechanism and key regulator of blood pressure' [84]. This is supported by the observation that in humans high blood pressure levels correlate with increased vertebral artery resistance $[84,85]$. Thus, could perfusion of the brain be a determinant of the set point of arterial pressure, and is brainstem hypoperfusion a contributing mechanism in neurogenic hypertension? In support of this, increasing cerebral artery vascular resistance activates the sympathetic nervous system in rats [77]. Further, Cates et al. have demonstrated that vertebral artery narrowing, increased vertebral artery resistance, a shift to anaerobic metabolism within the brainstem and an exaggerated sympatho-excitatory response to reduced cerebral perfusion, all occur prior the onset of hypertension in spontaneous hypertensive rats [86]. Tissue oxygen levels in the spontaneously hypertensive rat were found to be $15 \mathrm{mmHg}$ lower than in a normotensive rat at the same level of arterial pressure [87]. The transduction mechanism is unknown but could include a central detector of hypoxia, an 'intracranial baroreceptor', ion channels sensitive to blood flow or shear stress [86], and may involve ATP and lactate [87]. For example, both traumatic and nontraumatic brain injury can result in paroxysmal sympathetic hyperactivity and hypertension [88].

\section{Conclusion \& future perspective}

Although many antihypertensive drugs have a degree of penetrance into the brain, targeting the CNS with drugs causes poorly tolerated side effects and triggers a major clinical challenge of poor patient compliance to medication. According to our hypothesis, we need to consider targets within the PNS and identify 
those visceral afferents that have become sensitized and tonically activated in hypertension. Establishing the molecular mechanisms for this afferent tonicity would then allow selective drugs (that need not cross the blood-brain barrier) to temper their aberrant discharge. An ideal treatment would be a drug that targets a defined organ with known aberrant activity and blocks this pathological signaling while preserving the physiological operation of the afferent system. For example, selective blockade of purinergic receptor subtype (P2X3) abolished chronic pathological cough but defensive coughing (such as that evoked following aspiration) was preserved [89]. Reducing organ hypoxic-hypoperfusion and improving organ blood flow and oxygen reserve/capacity is another considered approach for treating hypertension. Experimental evidence shows that intrarenal RAS is compartmentalized from the systemic RAS such that intrarenal angiotensinconverting enzyme is not adequately inhibited by plasma concentrations of angiotensin-converting enzyme inhibition in currently used dosages [90]. Given that AngII can cause renal hypoxia and hypertension (see above), RAS inhibition selectively within the kidney may ameliorate hypoxic-hypoperfusion and reduce sympathetic hyperactivity. Furthermore, activation of HIF- $1 \alpha$ and HIF- $2 \alpha$ has been demonstrated to protect kidneys from hypoxia and progressive injury [91] by making the kidneys less sensitive to hypoxic-hypoperfusion. Notably, the efficacy of HIF activation appears to depend on when it is administered during hypertensive kidney disease [92] stressing the importance of timing relative to disease progression. Taken together, we propose that organ blood flow supply-demand mismatch could be a target for treating the sympathetic hyperactivity in hypertension. The future challenge is to offset arteriolar vasoconstriction, increase organ blood flow and replenish the blood flow and oxygen supply of specific bodily organs.

Financial \& competing interests disclosure This work was supported by the British Heart Foundation (no. FS/14/2/30630 and RG/12/6/29670), the Royal Society (RG140387) and the European Union, Seventh Framework Programme, Marie Curie Actions (CARPEDIEM no. 612280). The authors have no other relevant affiliations or financial involvement with any organization or entity with a financial interest in or financial conflict with the subject matter or materials discussed in the manuscript apart from those disclosed.

No writing assistance was utilized in the production of this manuscript.

Open access

This work is licensed under the Creative Commons Attribution 4.0 License. To view a copy of this license, visit http://creativecommons.org/licenses/by/4.0/

\section{EXECUTIVE SUMMARY}

- In physiological conditions, blood flow is distributed to all organs in proportion to local oxygen/energy demand.

- Blood flow demand can outstrip supply bringing about hypoxic-hypoperfusion especially when vasodilatory mechanisms are compromised.

- Aberrant afferent signaling from an organ may occur in response to hypoperfusion or unmet oxygen demand.

- Afferent sensitization can lead to excessive reflexly evoked sympathetic vasoconstriction, hypoxic-hypoperfusion and reduced blood flow that leads to further activation of afferent activity.

- Recent clinical trials have shown antihypertensive potency of bilateral renal nerve denervation in some drug-resistant hypertensive patients and carotid body afferent denervation in heart failure patients.

- Hypertension is associated with an exaggerated exercise pressor response and we propose sensitization and aberrant discharge from skeletal muscle afferents contribute to excessive sympathetic activity.

- The brain stem may detect blood flow directly, thereby acting as a CNS determinant of the set point of vasomotor sympathetic tone and contribute to sympathetic excess in the hypertensive condition if it becomes hypoperfused.

- We propose that organ blood flow supply-demand mismatch is a major source of visceral afferent drive and can extend across organs contributing to sympathetic excess and hypertension, a process that is exacerbated during nocturnal blood pressure dipping. 


\section{References}

Papers of special note have been highlighted as:

- of interest; $\bullet$ of considerable interest

1 Wolff C. Colloid supplementation during induction of anesthesia. Emerg. Med. 1(2), 34-38 (2015).

2 Mayet J, Hughes A. Cardiac and vascular pathophysiology in hypertension. Heart 89(9), 1104-1109 (2003).

3 Hart EC, Charkoudian N. Sympathetic neural regulation of blood pressure: influences of sex and aging. Physiology (Bethesda) 29(1), 8-15 (2014).

4 Esler M, Lambert E, Schlaich M. Point: chronic activation of the sympathetic nervous system is the dominant contributor to systemic hypertension. J. Appl. Physiol. (1985) 109(6), 1996-1998 (2010).

5 Hines T, Toney GM, Mifflin SW. Responses of neurons in the nucleus tractus solitarius to stimulation of heart and lung receptors in the rat. Circ. Res. 74(6), 1188-1196 (1994).

6 Foss JD, Wainford RD, Engeland WC, Fink GD, Osborn JW. A novel method of selective ablation of afferent renal nerves by periaxonal application of capsaicin. Am. J. Physiol. Regul. Integr. Comp. Physiol. 308(2), R112-R122 (2015).

- Describes an exciting new methodology to selectively abolish renal afferent nerves in rats.

7 Campese VM, Kogosov E. Renal afferent denervation prevents hypertension in rats with chronic renal failure. Hypertension 25 (4 Pt 2), 878-882 (1995).

- A classic paper on experimental renal denervation in hypertension and kidney disease.

8 Abdala AP, Mcbryde FD, Marina N et al. Hypertension is critically dependent on the carotid body input in the spontaneously hypertensive rat. J. Physiol. 590 (Pt 17), 4269-4277 (2012).

- Demonstrates that the carotid body is, in part, responsible for elevated sympathetic tone and critical for the genesis of hypertension in a hereditary hypertensive rat model.

9 Mcbryde FD, Abdala AP, Hendy EB et al. The carotid body as a putative therapeutic target for the treatment of neurogenic hypertension. Nat. Commun. 4, 2395 (2013).

-. Outlines the role and possible therapeutic target of the carotid body in neurogenic hypertension.

10 Niewinski P, Janczak D, Rucinski A et al. Carotid body removal for treatment of chronic systolic heart failure. Int. J. Cardiol. 168(3), 2506-2509 (2013).

11 Niewinski P, Janczak D, Rucinski A et al. Dissociation between blood pressure and heart rate response to hypoxia after bilateral carotid body removal in men with systolic heart failure. Exp. Physiol. 99(3), 552-561 (2014).

12 Movies and Graphics. www.moviesandgraphics.com

13 Joyner MJ, Casey DP. Regulation of increased blood flow (hyperemia) to muscles during exercise: a hierarchy of competing physiological needs. Physiol. Rev. 95(2), 549-601 (2015).

14 Zhao W, Swanson SA, Ye J et al. Reactive oxygen species impair sympathetic vasoregulation in skeletal muscle in angiotensin II-dependent hypertension. Hypertension 48(4), 637-643 (2006).

15 Thomas GD. Functional sympatholysis in hypertension. Auton. Neurosci. 188, 64-68 (2015).

- Classic paper on neural control of kidney function.

16 Hilton SM. Ways of viewing the central nervous control of the circulation - old and new. Brain Res. 87(2-3), 213-219 (1975).

17 Kopp UC, Cicha MZ, Smith LA, Mulder J, Hokfelt T. Renal sympathetic nerve activity modulates afferent renal nerve activity by PGE2-dependent activation of alpha1- and alpha2-adrenoceptors on renal sensory nerve fibers. Am. J. Physiol. Regul. Integr. Comp. Physiol. 293(4), R1561-R1572 (2007).

18 Schlereth T, Drummond PD, Birklein F. Inflammation in CRPS: role of the sympathetic supply. Auton. Neurosci. 182, 102-107 (2014).

19 Ford AP. In pursuit of P2X3 antagonists: novel therapeutics for chronic pain and afferent sensitization. Purinergic Signal. 8(Suppl. 1), 3-26 (2012).

20 Ives SJ, Amann M, Venturelli M et al. The mechanoreflex and hemodynamic response to passive leg movement in heart failure. Med. Sci. Sports Exerc. 48(3), 368-376 (2015).

21 Iversen BM, Kvam FI, Matre K, Ofstad J. Resetting of renal blood autoregulation during acute blood pressure reduction in hypertensive rats. Am. J. Physiol. 275(2 Pt 2), R343-R349 (1998).

22 Renna NF, De Las Heras N, Miatello RM. Pathophysiology of vascular remodeling in hypertension. Int. J. Hypertens. 2013, 808353 (2013).
23 Koroboki E, Manios E, Psaltopoulou T et al. Circadian variation of blood pressure and heart rate in normotensives, white-coat, masked, treated and untreated hypertensives. Hellenic J. Cardiol. 53(6), 432-438 (2012).

24 Sherwood A, Steffen PR, Blumenthal JA, Kuhn C, Hinderliter AL. Nighttime blood pressure dipping: the role of the sympathetic nervous system. Am. J. Hypertens. 15 (2 Pt 1), 111-118 (2002).

25 Johns EJ, Kopp UC, Dibona GF. Neural control of renal function. Compr. Physiol. 1(2), 731-767 (2011).

26 Kopp UC. Role of renal sensory nerves in physiological and pathophysiological conditions. Am. J. Physiol. Regul. Integr. Comp. Physiol. 308(2), R79-R95 (2015).

27 Katholi RE. Renal nerves and hypertension: an update. Fed. Proc. 44(13), 2846-2850 (1985).

28 Ye S, Ozgur B, Campese VM. Renal afferent impulses, the posterior hypothalamus, and hypertension in rats with chronic renal failure. Kidney Int. 51(3), 722-727 (1997).

29 Campese VM, Kogosov E, Koss M. Renal afferent denervation prevents the progression of renal disease in the renal ablation model of chronic renal failure in the rat. Am. J. Kidney Dis. 26(5), 861-865 (1995).

30 Kopp UC, Cicha MZ, Smith LA. Dietary sodium loading increases arterial pressure in afferent renal-denervated rats. Hypertension 42(5), 968-973 (2003).

31 Cowley AW, Jr., Abe M, Mori T, O'connor PM, Ohsaki Y, Zheleznova NN. Reactive oxygen species as important determinants of medullary flow, sodium excretion, and hypertension. Am. J. Physiol. Renal Physiol. 308(3), F179-F197 (2015).

32 Krum H, Schlaich M, Whitbourn R et al. Catheter-based renal sympathetic denervation for resistant hypertension: a multicentre safety and proof-of-principle cohort study. Lancet 373(9671), 1275-1281 (2009).

33 Esler MD, Krum H, Sobotka PA, Schlaich MP, Schmieder RE, Bohm M. Renal sympathetic denervation in patients with treatment-resistant hypertension (The Symplicity HTN-2 Trial): a randomised controlled trial. Lancet 376(9756), 1903-1909 (2010).

34 Schlaich MP, Hering D, Sobotka PA, Krum $\mathrm{H}$, Esler MD. Renal denervation in human hypertension: mechanisms, current findings, and future prospects. Curr. Hypertens. Rep. 14(3), 247-253 (2012).

- Important paper demonstrating that renal hypoxia precedes renal pathology. 
35 Bhatt DL, Kandzari DE, O'neill WW et al. A controlled trial of renal denervation for resistant hypertension. N. Engl. J. Med. 370(15), 1393-1401 (2014).

36 Salman IM. Cardiovascular autonomic dysfunction in chronic kidney disease: a comprehensive review. Curr. Hypertens. Rep. 17(8), 59 (2015).

37 Heyman SN, Khamaisi M, Rosen S, Rosenberger C. Renal parenchymal hypoxia, hypoxia response and the progression of chronic kidney disease. Am. J. Nephrol. 28(6), 998-1006 (2008).

38 Legrand M, Mik EG, Johannes T, Payen D, Ince $\mathrm{C}$. Renal hypoxia and dysoxia after reperfusion of the ischemic kidney. Mol. Med. 14(7-8), 502-516 (2008).

39 Tanaka T, Kato H, Kojima I et al. Hypoxia and expression of hypoxia-inducible factor in the aging kidney. J. Gerontol. A Biol. Sci. Med. Sci. 61(8), 795-805 (2006).

40 Manotham K, Tanaka T, Matsumoto M et al. Evidence of tubular hypoxia in the early phase in the remnant kidney model. J. Am. Soc. Nephrol. 15(5), 1277-1288 (2004).

41 Campese VM. A new model of neurogenic hypertension caused by renal injury: pathophysiology and therapeutic implications. Clin. Exp. Nephrol. 7(3), 167-171 (2003).

42 Evans RG, Gardiner BS, Smith DW, O'connor PM. Intrarenal oxygenation: unique challenges and the biophysical basis of homeostasis. Am. J. Physiol. Renal Physiol. 295(5), F1259-F1270 (2008).

43 Nangaku M. Chronic hypoxia and tubulointerstitial injury: a final common pathway to end-stage renal failure. J. Am. Soc. Nephrol. 17(1), 17-25 (2006).

44 Evans RG, Ince C, Joles JA et al. Haemodynamic influences on kidney oxygenation: clinical implications of integrative physiology. Clin. Exp. Pharmacol. Physiol. 40(2), 106-122 (2013).

45 Hansell P, Welch WJ, Blantz RC, Palm F. Determinants of kidney oxygen consumption and their relationship to tissue oxygen tension in diabetes and hypertension. Clin. Exp. Pharmacol. Physiol. 40 (2), 123-137 (2013).

46 Singh P, Ricksten SE, Bragadottir G, Redfors $B$, Nordquist L. Renal oxygenation and haemodynamics in acute kidney injury and chronic kidney disease. Clin. Exp. Pharmacol. Physiol. 40 (2), 138-147 (2013).

47 Liss P, Cox EF, Eckerbom P, Francis ST. Imaging of intrarenal haemodynamics and oxygen metabolism. Clin. Exp. Pharmacol. Physiol. 40(2), 158-167 (2013).
48 Nangaku M, Rosenberger C, Heyman SN, Eckardt KU. Regulation of hypoxia-inducible factor in kidney disease. Clin. Exp. Pharmacol. Physiol. 40(2), 148-157 (2013).

49 Kondo N, Kiyomoto H, Yamamoto T et al. Effects of calcium channel blockade on angiotensin II-induced peritubular ischemia in rats. J. Pharmacol. Exp. Ther. 316(3), 1047-1052 (2006).

50 Friederich-Persson M, Persson P, Fasching A, Hansell P, Nordquist L, Palm F. Increased kidney metabolism as a pathway to kidney tissue hypoxia and damage: effects of triiodothyronine and dinitrophenol in normoglycemic rats. Adv. Exp. Med. Biol. 789, 9-14 (2013).

51 Friederich-Persson M, Thorn E, Hansell P, Nangaku M, Levin M, Palm F. Kidney hypoxia, attributable to increased oxygen consumption, induces nephropathy independently of hyperglycemia and oxidative stress. Hypertension 62 (5), 914-919 (2013).

52 Hering D, Zdrojewski Z, Krol E et al. Tonic chemoreflex activation contributes to the elevated muscle sympathetic nerve activity in patients with chronic renal failure. J. Hypertens. 25(1), 157-161 (2007).

53 Marshall JM. Peripheral chemoreceptors and cardiovascular regulation. Physiol. Rev. 74(3), 543-594 (1994).

54 Blessing WW, Yu Y, Nalivaiko E. Medullary projections of rabbit carotid sinus nerve. Brain Res. 816(2), 405-410 (1999).

55 Schultz HD, Marcus NJ, Del Rio R. Role of the carotid body in the pathophysiology of heart failure. Curr. Hypertens. Rep. 15(4), 356-362 (2013).

56 Ding Y, Li YL, Schultz HD. Role of blood flow in carotid body chemoreflex function in heart failure. J. Physiol. 589(Pt 1), 245-258 (2011).

57 Barnett S, Mulligan E, Wagerle LC, Lahiri S Measurement of carotid body blood flow in cats by use of radioactive microspheres. J. Appl. Physiol. (1985) 65(6), 2484-2489 (1988).

58 Nobrega AC, O’leary D, Silva BM, Marongiu E, Piepoli MF, Crisafulli A. Neural regulation of cardiovascular response to exercise: role of central command and peripheral afferents. BioMed Res. Int. 2014, 478965 (2014).

59 Li J, Xing J. Muscle afferent receptors engaged in augmented sympathetic responsiveness in peripheral artery disease. Front. Physiol. 3, 247 (2012).

60 Fisher JP, Seifert T, Hartwich D, Young CN, Secher NH, Fadel PJ. Autonomic control of heart rate by metabolically sensitive skeletal muscle afferents in humans. J. Physiol. 588(Pt 7), 1117-1127 (2010).

61 Li J, Sinoway LI. ATP stimulates chemically sensitive and sensitizes mechanically sensitive afferents. Am. J. Physiol. Heart Circ. Physiol. 283(6), H2636-H2643 (2002).

62 Berger A, Grossman E, Katz M et al. Exercise blood pressure and the risk for future hypertension among normotensive middleaged adults. J. Am. Heart Assoc. 4(4), pii: e001710 (2015).

63 Wang HJ, Li YL, Gao L, Zucker IH, Wang W. Alteration in skeletal muscle afferents in rats with chronic heart failure. J. Physiol. 588(Pt 24), 5033-5047 (2010).

64 Vongpatanasin W, Wang Z, Arbique D et al. Functional sympatholysis is impaired in hypertensive humans. J. Physiol. 589(Pt 5), 1209-1220 (2011).

65 Mortensen SP, Saltin B. Regulation of the skeletal muscle blood flow in humans. Exp. Physiol. 99(12), 1552-1558 (2014).

66 Rosenmeier JB, Hansen J, Gonzalez-Alonso J. Circulating ATP-induced vasodilatation overrides sympathetic vasoconstrictor activity in human skeletal muscle. J. Physiol. 558(Pt 1), 351-365 (2004).

67 Nishiyama A, Fujisawa Y, Fukui T et al. Role of nitric oxide in regional blood flow in angiotensin II-induced hypertensive rats. Hypertens. Res. 24(4), 421-427 (2001).

68 Hellsten Y, Nyberg M, Jensen LG, Mortensen SP. Vasodilator interactions in skeletal muscle blood flow regulation. J. Physiol. 590 (Pt 24), 6297-6305 (2012)

69 Koba S, Watanabe R, Kano N, Watanabe T. Oxidative stress exaggerates skeletal muscle contraction-evoked reflex sympathoexcitation in rats with hypertension induced by angiotensin II. Am. J. Physiol. Heart Circ. Physiol. 304(1), H142-H153 (2013).

70 Nishiyama A, Fukui T, Fujisawa Y et al. Systemic and regional hemodynamic responses to tempol in angiotensin II-infused hypertensive rats. Hypertension 37(1), 77-83 (2001).

71 Zhao W, Swanson SA, Ye J et al. Reactive oxygen species impair sympathetic vasoregulation in skeletal muscle in angiotensin II-dependent hypertension. Hypertension 48(4), 637-643 (2006).

72 Pan HL, Deal DD, Xu Z, Chen SR. Differential responses of regional sympathetic activity and blood flow to visceral afferent stimulation. Am. J. Physiol. Regul. Integr. Comp. Physiol. 280(6), R1781-R1789 (2001). 
73 Sartor DM. Sympathoinhibitory signals from the gut and obesity-related hypertension. Clin. Auton. Res. 23(1), 33-39 (2013).

74 Prewitt RL, Rice DC, Dobrian AD. Adaptation of resistance arteries to increases in pressure. Microcirculation 9(4), 295-304 (2002).

75 Reid JL. Vascular reactivity, adrenergic mechanisms, and arteriolar resistance in hypertension. J. Cardiovasc. Pharmacol. 12(Suppl. 3), S114-S120 (1988).

76 Mayhan WG, Werber AH, Heistad DD. Protection of cerebral vessels by sympathetic nerves and vascular hypertrophy. Circulation 75(1 Pt 2), I107-I112 (1987).

77 Baumbach GL, Heistad DD, Siems JE. Effect of sympathetic nerves on composition and distensibility of cerebral arterioles in rats. J. Physiol. 416, 123-140 (1989).

78 Cates MJ, Steed PW, Abdala AP, Langton PD, Paton JF. Elevated vertebrobasilar artery resistance in neonatal spontaneously hypertensive rats. J. Appl. Physiol. (1985) 111(1), 149-156 (2011).

79 Jensen KJ, Alpini G, Glaser S. Hepatic nervous system and neurobiology of the liver. Compr. Physiol. 3(2), 655-665 (2013).

80 Gao L, Zhu Z, Zucker IH, Wang W. Cardiac sympathetic afferent stimulation impairs baroreflex control of renal sympathetic nerve activity in rats. Am. J. Physiol. Heart Circ. Physiol. 286(5), H1706-H1711 (2004).
81 Kennedy C. ATP as a cotransmitter in the autonomic nervous system. Auton. Neurosci. 191, 2-15 (2015).

82 Gibbs GF, Drummond PD, Finch PM, Phillips JK. Unravelling the pathophysiology of complex regional pain syndrome: focus on sympathetically maintained pain. Clin. Exp. Pharmacol. Physiol. 35(7), 717-724 (2008).

83 Schultz HD, Li YL, Ding Y. Arterial chemoreceptors and sympathetic nerve activity: implications for hypertension and heart failure. Hypertension 50 (1), 6-13 (2007).

84 Paton JF, Dickinson CJ, Mitchell G. Harvey Cushing and the regulation of blood pressure in giraffe, rat and man: introducing 'Cushing's mechanism'. Exp. Physiol. 94(1), 11-17 (2009)

-• Outlines the Cushing's mechanism hypothesis.

85 Dickinson CJ, Thomson AD. A post mortem study of the main cerebral arteries with special reference to their possible role in blood pressure regulation. Clin. Sci. 19, 513-538 (1960).

86 Cates MJ, Dickinson CJ, Hart EC, Paton JF. Neurogenic hypertension and elevated vertebrobasilar arterial resistance: is there a causative link? Curr. Hypertens. Rep. 14(3), 261-269 (2012).

87 Marina N, Ang R, Machhada A et al. Brainstem hypoxia contributes to the development of hypertension in the spontaneously hypertensive rat. Hypertension 65(4), 775-783 (2015).

88 Baguley IJ, Perkes IE, Fernandez-Ortega JF, Rabinstein AA, Dolce G, Hendricks HT.

Paroxysmal sympathetic hyperactivity after acquired brain injury: consensus on conceptual definition, nomenclature, and diagnostic criteria. J. Neurotrauma 31(17), 1515-1520 (2014)

89 Abdulqawi R, Dockry R, Holt K et al. P2X3 receptor antagonist (AF-219) in refractory chronic cough: a randomised, double-blind, placebo-controlled Phase 2 study. Lancet 385(9974), 1198-1205 (2015).

90 Nishiyama A, Seth DM, Navar LG. Renal interstitial fluid concentrations of angiotensins I and II in anesthetized rats. Hypertension 39(1), 129-134 (2002).

91 Tanaka T, Nangaku M. Drug discovery for overcoming chronic kidney disease (CKD): prolyl-hydroxylase inhibitors to activate hypoxia-inducible factor (HIF) as a novel therapeutic approach in CKD. J. Pharmacol. Sci. 109(1), 24-31 (2009).

92 Yu X, Fang Y, Liu H et al. The balance of beneficial and deleterious effects of hypoxiainducible factor activation by prolyl hydroxylase inhibitor in rat remnant kidney depends on the timing of administration. Nephrol. Dial. Transplant. 27(8), 3110-3119 (2012). 